\title{
Susceptibility-Diffusion Mismatch Predicts Thrombolytic Outcomes: A Retrospective Cohort Study
}

M. Lou, Z. Chen, J. Wan, H. Hu, X. Cai, Z. Shi, and J. Sun

\begin{abstract}
BACKGROUND AND PURPOSE: Asymmetric hypointensity of cerebral veins on susceptibility-weighted imaging has been shown to indirectly reflect tissue hypoxia after cerebral ischemia. We therefore investigated whether patients with prominent asymmetry of the cerebral veins on SWI and a relatively small diffusion-weighted imaging lesion (SWI-DWI mismatch), representing the presence of salvageable tissue, were more likely to benefit from thrombolytic therapy.
\end{abstract}

MATERIALS AND METHODS: We conducted a retrospective study of the anterior circulation of patients with ischemic stroke with SWI/DWI acquired before thrombolysis. The asymmetry index was defined as the ratio of cerebral vein voxel count between the ischemic and normal hemisphere on the SWI phase map. We defined SWI-DWI mismatch as an asymmetry index score of $\geq 1.75$ with a DWI lesion volume of $\leq 25 \mathrm{~mL}$. Favorable outcome was defined as modified Rankin Scale $0-2$ at 3 months. Univariate and multivariate logistic regression analyses were used to examine the association between the mismatch profile and favorable outcome.

RESULTS: Fifty-four patients undergoing thrombolytic treatment were enrolled in this study. The rate of favorable outcome was significantly higher among patients with baseline SWI-DWI mismatch compared with those without (78\% versus $44 \%$; adjusted odds ratio, 6.317 ; $95 \% \mathrm{Cl}, 1.12-35.80 ; P=.037)$. Patients with SWI-DWI mismatch were also more likely to have a favorable outcome from reperfusion (91\% versus $43 \%, P=.033)$ or recanalization $(100 \%$ versus $40 \%, P=.013)$. The accuracy of SWI-DWI mismatch for predicting favorable outcome was higher than that of perfusion-diffusion mismatch (63\% versus $48.1 \%$ ).

CONCLUSIONS: The presence of SWI-DWI mismatch may identify patients with ischemia who would benefit from early reperfusion therapy.

ABBREVIATIONS: $\mathrm{Al}=$ asymmetry index; $\mathrm{TIMI}=$ Thrombolysis in Myocardial Infarction; Tmax $=$ time-to-peak of the residue function

ntravenous thrombolysis with recombinant tissue plasminogen

activator is a proved treatment for acute ischemic stroke within 4.5 hours of symptom onset. ${ }^{1}$ However, increasing evidence indicates that it may be more logical to move from this time-based method to a tissue-based imaging paradigm for patient selection

Received January 17, 2014; accepted after revision March 23.

From the Departments of Neurology (M.L., Z.C., J.W., H.H., X.C., Z.S.) and Radiology (J.S.), the Second Affiliated Hospital of Zhejiang University, School of Medicine, Hangzhou, China.

This work was supported by the Science Technology Department of Zhejiang Province (grant 2013C13G2010032), Zhejiang Provincial Natural Science Foundation of China (grant LRI2H09001), and the National Natural Science Foundation of China (grant 81171095).

Please address correspondence to Jianzhong Sun, MB, Department of Neurology, e-mail: hzzjsjz@163.com; or Min Lou, MD, PhD, Department of Radiology, the Second Affiliated Hospital of Zhejiang University School of Medicine, 88 Jiefang Rd, Hangzhou, China; e-mail: loumingxc@vip.sina.com

- Indicates open access to non-subscribers at www.ajnr.org

三 Indicates article with supplemental on-line tables.

http://dx.doi.org/10.3174/ajnr.A4017 for thrombolytic therapy because carefully selected patients may still benefit from thrombolytic therapy beyond 4.5 hours. ${ }^{2}$ The perfusion-diffusion mismatch model, based on a mismatch between the lesion volume on MR perfusion imaging and diffusionweighted imaging, has been proposed as a method of selecting patients for reperfusion therapy. Despite theoretic evidence, there is no consensus about the optimal perfusion parameter to accurately define ischemic tissue, and it is uncertain whether perfusion lesion volumes overestimate the penumbra tissue. ${ }^{3,4}$ Positronemission tomography is considered the criterion standard for detection of reversibly damaged tissue. However, its availability is limited to a few centers and restricted by complex logistics. ${ }^{5}$ Thus, until now, the optimal method to identify eligible candidates for reperfusion therapy in the acute setting remains elusive.

More recently, susceptibility-weighted imaging, which was originally called blood oxygen level-dependent venographic imaging, has demonstrated advantages over conventional gradientecho $\mathrm{T} 2{ }^{\star}$-weighted imaging in the detection of hemorrhagic events due to its exquisite sensitivity to paramagnetic substances 
such as deoxyhemoglobin. ${ }^{6}$ SWI venography allows clear visualization of cerebral veins. ${ }^{7}$ Shortly after arterial occlusion in patients with acute stroke, there is an increase in deoxyhemoglobin and a decrease in oxyhemoglobin within cerebral veins and tissue capillaries, due to an increase in the oxygen extraction fraction. ${ }^{8-10}$ This intravascular deoxygenation leads to a signal drop along the course of cerebral veins on SWI venography. ${ }^{1-14}$ SWI venography may thus provide the oxygen metabolic information about ischemic brain tissue by the noninvasive estimation of blood oxygen level-dependent levels.

A previous study by using gradient-echo $\mathrm{T} 2{ }^{\star}$-weighted imaging demonstrated that abnormal hypointensity of the deep cerebral veins was correlated with a delayed mean transit time and a large perfusion defect. ${ }^{9}$ Abnormal hypointensity of superficial cerebral veins also correlates with increased cerebral blood volume. ${ }^{15}$ Due to the relatively poor resolution of gradient-echo T2* ${ }^{*}$ weighted imaging, no previous studies combined the deep and superficial venous systems as a whole when examining the clinical implications of hypointense cerebral veins. The higher resolution of SWI allowed us to evaluate the ischemic state of the whole brain and the level of tissue hypoxia caused by hypoperfusion. We then developed a novel mismatch model, based on prominent hypointense cerebral veins on SWI and a relatively small DWI lesion (SWI-DWI mismatch), and tested whether this model was more likely to benefit from thrombolytic therapy.

\section{MATERIALS AND METHODS \\ Patients}

We retrospectively reviewed our prospectively collected data base for consecutive patients with acute ischemic stroke who received intravenous $\mathrm{rtPA}$ (alteplase, $0.9 \mathrm{mg} / \mathrm{kg}$, up to a maximum of 90 $\mathrm{mg} ; 10 \%$ of the total dosage as a bolus and the rest during 1 hour) within 6 hours after symptom onset from August 2009 to December 2012. Our predefined inclusion criteria for MR imagingguided intravenous thrombolysis included the following: 1) age older than 18 years; 2) stroke symptoms lasting $>1$ hour; 3) an NIHSS score of $\geq 4$ or NIHSS score of $<4$ but with aphasia or severe dysarthria; 4) the absence of intracranial hemorrhage confirmed by SWI; 5) onset-to-treatment time within 4.5 hours and fulfilling standard clinical criteria, or onset-to-treatment time of 4.5-6 hours with large-vessel occlusion on MR angiography and fulfilling the perfusion-diffusion mismatch criteria: DWI lesion of $\leq 70 \mathrm{~mL}$, a perfusion lesion to diffusion lesion volume ratio of $\geq 1.2$, and an absolute difference between perfusion and diffusion lesion volume of $>10 \mathrm{~mL} .{ }^{16}$ Standard IV thrombolysis exclusion criteria were applied according to current guidelines. ${ }^{17} \mathrm{We}$ prospectively obtained baseline demographic and clinical information. All subjects gave written informed consent before the study, and the protocols were approved by the human ethics committee of the hospital. All procedures were conducted according to the principles expressed in the Declaration of Helsinki.

\section{MR Imaging Protocol}

Multimodal MR imaging was performed on a 3T system (Signa Excite HD; GE Healthcare, Milwaukee, Wisconsin) before initiation of thrombolysis. Structural imaging included DWI $(\mathrm{TR}=$ $4000 \mathrm{~ms}, \mathrm{TE}=69.3 \mathrm{~ms}$, b-value $=1000 \mathrm{~s} / \mathrm{mm}^{2}$, acquisition ma- trix $=160 \times 160$, FOV $=240 \mathrm{~mm}$, section thickness $=5 \mathrm{~mm}$, section gap $=1 \mathrm{~mm}$, duration $=32$ seconds), a perfusionweighted sequence $(\mathrm{TR} / \mathrm{TE}=1500 / 30 \mathrm{~ms}$, acquisition matrix $=$ $128 \times 128$, dynamic scans $=50, \mathrm{FOV}=240 \mathrm{~mm}$, section thickness $=5 \mathrm{~mm}$, section gap $=1 \mathrm{~mm}$, gadolinium dose $=15 \mathrm{~mL}$, contrast speed $=4-5 \mathrm{~mL} / \mathrm{s}$, duration $=1$ minute 15 seconds), time-of-flight MR angiography (TR/TE/flip angle $=20 \mathrm{~ms} / 3.2 \mathrm{~ms} /$ $15^{\circ}$, acquisition matrix $=320 \times 224$, section thickness $=1.4 \mathrm{~mm}, 3$ slabs, duration $=3$ minutes 46 seconds), and SWI (3D multiecho $\mathrm{T} 2^{\star}$-weighted gradient-echo sequence, TR/TE/flip angle $=58 \mathrm{~ms} / 5.0$ $\mathrm{ms} / 20^{\circ}, \mathrm{FOV}=240 \mathrm{~mm}$, acquisition matrix $=384 \times 32$, section thickness $=2 \mathrm{~mm}$, duration $=3$ minutes 27 seconds). The entire duration of the MR imaging protocol was 15 minutes.

\section{Follow-Up Protocol and Clinical Assessments}

All patients experiencing thrombolysis underwent follow-up multimodal MR imaging within 24 hours after treatment. Clinical follow-up assessments included the NIHSS score at 1, 4, and 24 hours, 7 days, and 1 month and modified Rankin Scale scores at 1 and 3 months. Early NIHSS improvement was defined as an increase on the NIHSS of at least 8 points between baseline and 1 week after onset or an NIHSS score $=0$ at 1 week. ${ }^{18}$ Favorable outcome was defined as mRS $0-2$ at 3 months.

\section{Radiologic Assessment}

DWI lesion volumes were measured by using a semiautomated thresholding algorithm that identified the region with intensity higher than that in the contralateral frontal lobe by $>3$ SDs. ${ }^{19}$ Artifactual lesion areas were visually identified by an experienced neuroradiologist (J.S.) and were manually removed. Time-to-peak of the residue function (Tmax) maps were generated by deconvolution of the tissue concentration-time curve by using an arterial input function from the contralateral middle cerebral artery. ${ }^{19,20}$ The perfusion lesion was defined as a Tmax delay of $>6$ seconds. ${ }^{21}$ Reperfusion was defined as a $\geq 50 \%$ and $\geq 10 \mathrm{~mL}$ reduction of PWI lesion volume ${ }^{22}$ between baseline and follow-up within 24 hours of MR imaging. PWI lesions of $\leq 3 \mathrm{~mL}$ were excluded. ${ }^{23}$

The MRA was rated independently by 3 experienced stroke neurologists (M.L, Z.C., and J.S.), who then reached consensus by using the Thrombolysis in Myocardial Infarction (TIMI) grading scale $^{16,24}: 0=$ complete occlusion, which was defined as lack of flow signal of a vascular segment and distal vessels; $1=$ severe stenosis, defined as severe or critical stenosis of a vascular segment with significant reduction of flow signal distal to stenosis; $2=$ mild-to-moderate stenosis, which was characterized by stenosis with normal distal flow signal; 3 = normal arterial caliber. Recanalization was defined as improvement of TIMI grading by $\geq 2$ points from baseline to follow-up MRA.

\section{Definition of the Asymmetry Index}

We used a high-pass filter with a central matrix size of $32 \times 32$ to remove background field inhomogeneities to create the corrected SWI phase images. We used MRIcro software (http://www. mccauslandcenter.sc.edu/mricro/mricro/mricro.html) to assess venous structures in 5 consecutive sections around the level of the lateral ventricles (from immediately above the basal ganglia to the highest section including the ventricle) because these sections in- 

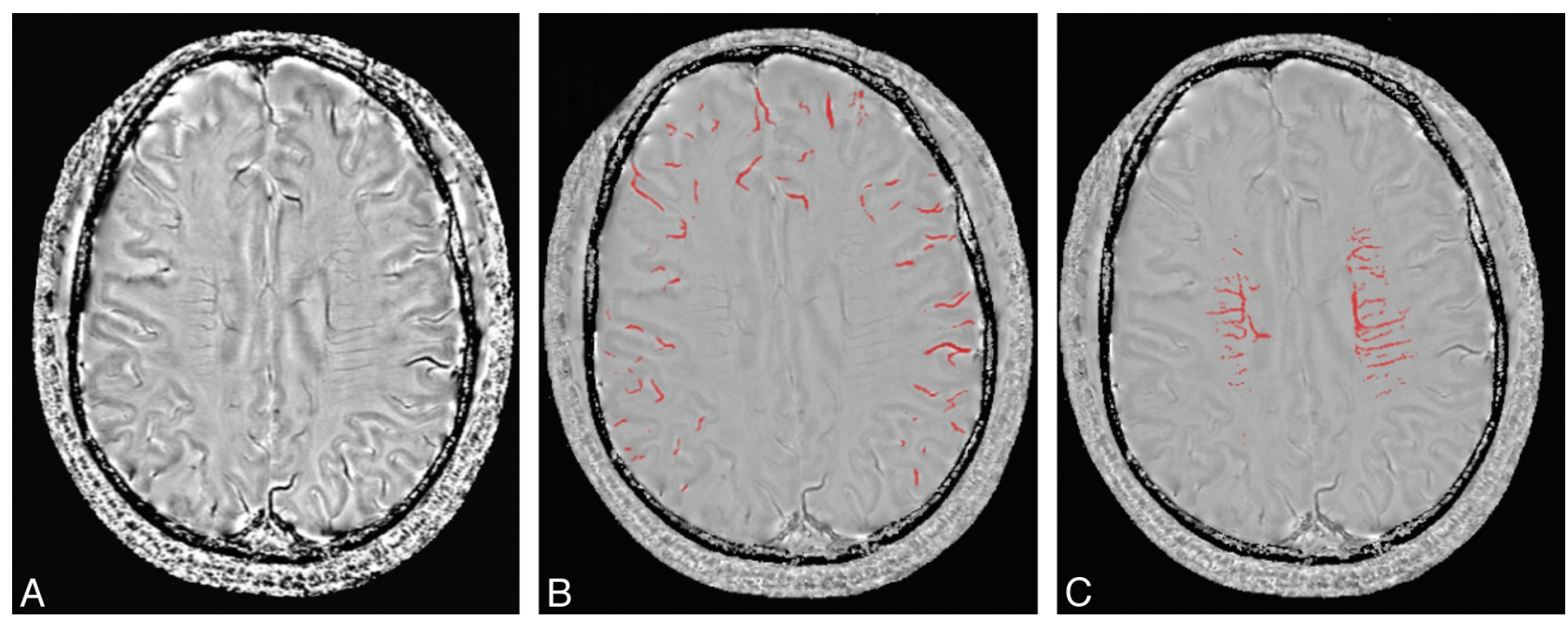

FIG 1. Image postprocessing and segmentation of cerebral venous structures based on SWI phase images. The SWI phase image ( $A$ ) was used to calculate the number of voxels of superficial cerebral veins $(B)$ and deep cerebral veins $(C)$ for quantification after segmentation. The asymmetry index of cerebral veins was set as a ratio of voxel numbers of cerebral veins between the ipsilateral and contralateral side.

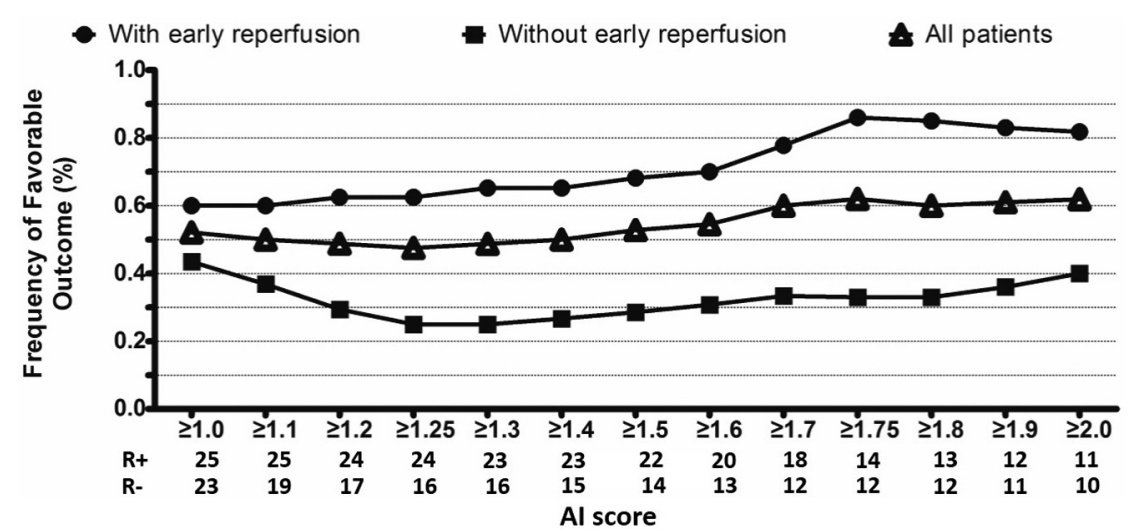

FIG 2. The Al score was positively correlated with the frequency of favorable outcome in patients with early reperfusion. The optimal Al score was set as 1.75 based on maximizing the Youden index (sensitivity, 83\%; specificity, 67\%; Youden index, 53\%) for predicting favorable outcome in patients with early reperfusion. $R(+)$ indicates the number of patients with early reperfusion; $\mathrm{R}(-)$, the number of patients without early reperfusion.

clude most of the cerebral veins draining the anterior circulation territory. The segmentation threshold for deep and superficial cerebral veins was determined as 2 SDs in voxel intensity below the mean of the fitted distribution of white matter and cortex, respectively (Fig 1). Bilateral venous voxel counts were computed, and an asymmetry index (AI) was defined as the ratio of the venous voxel number between the ischemic and normal hemispheres to eliminate individual bias. ${ }^{25-27}$

\section{Definition of SWI-DWI Mismatch}

The interaction among early reperfusion, AI score, and favorable outcome was assessed (Fig 2), and receiver operating characteristic analysis was used to determine the cutoff value for an optimal AI score when it gave the highest Youden index. ${ }^{20}$ We defined SWI-DWI mismatch as an AI score greater than the optimal threshold and a DWI lesion of $\leq 25 \mathrm{~mL} .^{28,29}$ To examine whether patients with SWI-DWI mismatch were more likely to benefit from reperfusion or recanalization after rtPA therapy, we compared the rate of favorable outcome from reperfusion or recanalization between patients with and without SWI-DWI mismatch.
We also compared the accuracy of this novel SWI-DWI mismatch with the perfusion-diffusion mismatch. We used several different perfusion-diffusion mismatch definitions: 1) standard PWI-DWI mismatch: mismatch ratio of $\geq 1.2$ and absolute mismatch of $\geq 10 \mathrm{~mL}^{16}$; 2 ) optimal PWI-DWI mismatch: mismatch ratio of $\geq 2.6$ and absolute mismatch of $\geq 10$ $\mathrm{mL}^{20}$; 3) target PWI-DWI mismatch: mismatch ratio $\geq 1.2$, absolute mismatch $\geq 10 \mathrm{~mL}$, Tmax $\geq 8$ seconds, lesion $\leq 100 \mathrm{~mL}$, and DWI $\leq 100 \mathrm{~mL} \cdot{ }^{30}$

\section{Statistical Analysis}

We divided patients into high and low AI groups, by using the Youden-derived threshold. We also compared variables between patients with and without SWI-DWI mismatch. We used the Fisher exact test to compare dichotomous variables between groups, the $t$ test for normally distributed continuous variables, and the Wilcoxon rank sum test for ordinal variables and nonnormally distributed continuous variables. Univariate and multivariate logistic regression analyses were used to investigate whether SWI-DWI mismatch was an independent predictor of favorable outcome. Statistical significance was set at $P<.05$. All statistical analyses were performed by using SPSS, Version 19 (IBM, Armonk, New York).

\section{RESULTS}

Eighty consecutive patients had multimodal MR imaging, and 60 had cerebral infarction in the anterior circulation territory. Six patients were excluded before the analysis because of poor-quality SWI due to dental artifacts ( 1 patient) and motion artifacts (5 patients), which interfered with the assessment of cerebral veins. Thus 54 patients were included in the analysis. The mean age of the patients was $69 \pm 13$ years, and the median baseline NIHSS 
Table 1: Univariate analysis of the baseline variables according to the asymmetry index of cerebral veins $^{\mathrm{a}}$

\begin{tabular}{|c|c|c|c|}
\hline Variable & High Al $(n=27)$ & Low AI $(n=27)$ & $P$ Value \\
\hline Mean age (yr) & $69 \pm 13$ & $69 \pm 14$ & .926 \\
\hline Sex, female (\%) & $8(30 \%)$ & $13(48 \%)$ & .264 \\
\hline Serum glucose (mmol/L) & $141 \pm 41$ & $159 \pm 72$ & .268 \\
\hline Systolic BP (mm Hg) & $156 \pm 19$ & $153 \pm 24$ & .653 \\
\hline Time to imaging (min) & $175 \pm 58$ & $186 \pm 68$ & .514 \\
\hline Time to treatment (min) & $222 \pm 68$ & $235 \pm 60$ & .448 \\
\hline Baseline NIHSS score & $15(6-18)$ & $9(5-14)$ & .096 \\
\hline \multicolumn{4}{|l|}{ Stroke risk factors } \\
\hline Atrial fibrillation (\%) & $13(48 \%)$ & $11(41 \%)$ & .785 \\
\hline Hypertension (\%) & $20(74 \%)$ & $18(67 \%)$ & .766 \\
\hline Diabetes mellitus (\%) & $7(26 \%)$ & $9(33 \%)$ & .766 \\
\hline Hyperlipidemia (\%) & $11(41 \%)$ & $9(33 \%)$ & .779 \\
\hline Current smoking (\%) & $11(41 \%)$ & $4(15 \%)$ & .066 \\
\hline Coronary heart disease (\%) & $7(26 \%)$ & $8(30 \%)$ & 1.000 \\
\hline Hyperhomocysteinemia (\%) & $9(33 \%)$ & $7(26 \%)$ & .766 \\
\hline White blood cell $/ \mathrm{mm}^{3}$ & $7.1 \pm 2.2$ & $8.3 \pm 2.4$ & .052 \\
\hline PT (sec) & $12.7 \pm 0.9$ & $12.4 \pm 1.4$ & .368 \\
\hline APTT (sec) & $27.6 \pm 5.6$ & $25.3 \pm 3.4$ & .066 \\
\hline INR & $1.06 \pm 0.08$ & $1.03 \pm 0.12$ & .325 \\
\hline Fibrinogen level (g/L) & $7.7 \pm 24.9$ & $2.8 \pm 0.9$ & .313 \\
\hline Serum creatinine level (mg/dL) & $0.82 \pm 0.14$ & $0.99 \pm 0.93$ & .366 \\
\hline Serum urea nitrogen level $(\mathrm{mg} / \mathrm{dL})$ & $16.2 \pm 5.1$ & $16.1 \pm 4.3$ & .909 \\
\hline TIMI grading & $1(0-1)$ & $2(0-3)$ & $.002^{\mathrm{b}}$ \\
\hline Sites of occlusion & & & $.042^{\mathrm{b}}$ \\
\hline Proximal & 22 & 14 & \\
\hline Distal & 5 & 13 & \\
\hline DWI lesion volume (mL) & $37 \pm 57$ & $15 \pm 33$ & .091 \\
\hline PWI lesion volume (mL) & $120 \pm 83$ & $58 \pm 67$ & $.004^{\mathrm{b}}$ \\
\hline 24-Hour DWI lesion volume (mL) & $66 \pm 82$ & $35 \pm 69$ & .131 \\
\hline Hemorrhagic transformation (\%) & $11(41 \%)$ & $7(26 \%)$ & .387 \\
\hline $\mathrm{HI}$ & $7(26 \%)$ & $5(19 \%)$ & .745 \\
\hline $\mathrm{PH}$ & $4(15 \%)$ & $2(33 \%)$ & .669 \\
\hline $\mathrm{slCH}$ & $1(4 \%)$ & $2(7 \%)$ & 1.000 \\
\hline Favorable outcome & 17 (63\%) & $13(48 \%)$ & .412 \\
\hline
\end{tabular}

Note:-BP indicates blood pressure; PT, prothrombin time; APTT, activated partial thromboplastin time; INR, international normalized ratio; $\mathrm{HI}$, hemorrhagic infarction; $\mathrm{PH}$, parenchymal hematoma; $\mathrm{slCH}$, symptomatic intracerebral hemorrhage.

${ }^{a}$ Table cells express results in mean \pm SD for normally distributed continuous variables, $n(\%)$ for dichotomous variables, and median (interquartile range) for ordinal variables and non-normally distributed continuous variables, respectively.

$\mathrm{b} p<.05$.

score was 12 (interquartile range, 5-17). Eight patients received thrombolytic therapy beyond 4.5 hours according to the criteria described in "Materials and Methods." Favorable outcome occurred in 3/3 with SWI-DWI mismatch versus $1 / 5$ (20\%) without SWI-DWI mismatch.

\section{Optimal AI Score for SWI-DWI Mismatch Analysis}

As shown in Fig 2, in the presence of reperfusion, the rate of favorable outcome correlated with increasing AI score. Receiver operating characteristic analysis revealed that the optimal threshold for the AI score was 1.75 (sensitivity, 86\%; specificity, 67\%; Youden index, 53\%) for predicting favorable outcome from early reperfusion.

\section{Al Score and Hypoperfusion Lesion Volume}

Table 1 summarizes the baseline and 24-hour follow-up variables in patients with high $(n=27)$ versus low AI scores $(n=27)$. Univariate analysis showed that patients with high AI scores had more frequent arterial occlusion (median TIMI 1 [interquartile range, $0-1$ ] versus 2 [interquartile range, $0-3$ ] , $P=.002$ ) and larger perfusion lesion volume $(120 \pm 83$ mL versus $58 \pm 67 \mathrm{~mL}, P=.004)$ than those with a low AI score. Figure 3 illustrates the relationship among AI score, perfusion lesion volume, and TIMI grading.

\section{SWI-DWI Mismatch and Favorable Outcome}

SWI-DWI mismatch, defined by a high AI score (AI $\geq 1.75)$ with a small DWI lesion volume $(\leq 25 \mathrm{~mL})$, was present in 18 of 54 patients (33\%). Univariate analysis showed that patients with SWIDWI mismatch were more likely to have early NIHSS improvement (67\% versus $31 \%, P=.019)$ and favorable outcome (On-line Table 1) compared with those without SWI-DWI mismatch (78\% versus $44 \%, P=.024)$. The rates of reperfusion, recanalization, and hemorrhagic transformation were not significantly different between these 2 groups. Logistic regression analysis revealed that the presence of SWI-DWI mismatch (OR = 6.317; 95\% CI, 1.12-35.80; $P=.037$ ) was independently associated with favorable outcome (On-line Table 2).

\section{Favorable Outcome of SWI-DWI Mismatch based on Reperfusion}

Table 2 shows that 11 patients $(61 \%)$ with SWI-DWI mismatch and 14 (39\%) patients without it achieved reperfusion. Patients with SWI-DWI mismatch were more likely to obtain favorable outcome from reperfusion than those without SWI-DWI mismatch $(91 \%$ versus $43 \%, P=.033)$.

\section{Comparison of Accuracy between SWI-DWI Mismatch and PWI-DWI Mismatch}

As shown in Table 3, the accuracy of SWI-DWI mismatch (63.0\%) was higher than that of PWI-DWI mismatch $37.0 \%$ for standard PWI-DWI mismatch, $44.4 \%$ for optimal PWI-DWI mismatch, $48.1 \%$ for target PWI-DWI mismatch).

\section{DISCUSSION}

Our study proposes a novel SWI-DWI mismatch paradigm for predicting neurologic outcome after intravenous thrombolysis. Patients with SWI-DWI mismatch had a higher rate of favorable outcome than those without this mismatch (78\% versus $44 \%$ ). The accuracy of SWI-DWI mismatch was higher than that of PWI-DWI mismatch in the current study. Thus, SWI-DWI mismatch may provide an alternative method for selecting patients who are good candidates for thrombolytic therapy.

Deoxyhemoglobin is used as an endogenous contrast agent due to its paramagnetic properties on SWI. Because venous blood volume contains higher concentrations of deoxyhemoglobin, the 


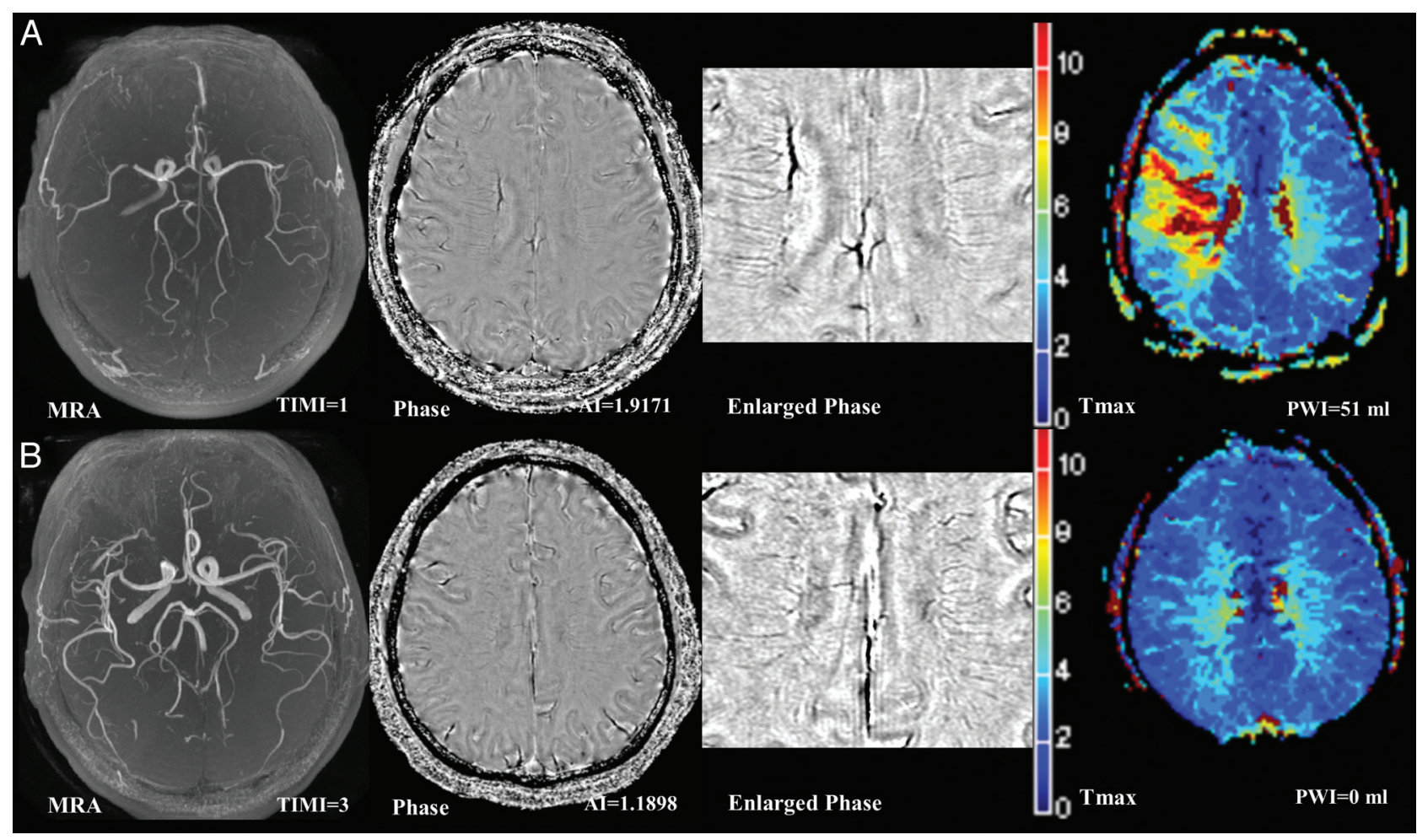

FIG 3. Representative pretreatment MR imaging in patients receiving thrombolysis. The first column on the left depicts time-of-flight MR angiography; the second and third columns depict phase images of susceptibility-weighted imaging and an enlarged view focusing on the deep cerebral veins. The right column depicts Tmax maps of perfusion-weighted MR imaging. Patients with a high $\mathrm{Al}$ score $(A, \mathrm{TIMI}=1, \mathrm{Al}$ score $=1.92$, $\mathrm{PWI}$ lesion volume $=51 \mathrm{~mL}$ ) had a lower TIMI score and larger PWI lesion volume than those with low Al scores $(B, \mathrm{TIMI}=3, \mathrm{Al}$ score $=1.19$, PWI lesion volume $=0 \mathrm{~mL}$ ).

Table 2: Different outcomes in patients with or without SWI-DWI mismatch based on reperfusion

\begin{tabular}{|c|c|c|c|c|}
\hline & \multicolumn{2}{|c|}{ Reperfusion } & \multicolumn{2}{|c|}{ No Reperfusion } \\
\hline & Total & $\begin{array}{l}\text { Favorable } \\
\text { Outcome }\end{array}$ & Total & $\begin{array}{l}\text { Favorable } \\
\text { Outcome }\end{array}$ \\
\hline SWI-DWI Mismatch $(n=18)$ & 11 & $10(91 \%)$ & 7 & $4(57 \%)$ \\
\hline $\begin{array}{l}\text { Non-SWI-DWI mismatch } \\
(n=36)\end{array}$ & 14 & $6(43 \%)$ & 22 & $10(45 \%)$ \\
\hline$P$ value & & .033 & & .682 \\
\hline
\end{tabular}

Table 3: The sensitivity, specificity, and accuracy between SWIDWI and PWI-DWI mismatches ${ }^{\mathrm{a}}$

\begin{tabular}{lcccl}
\hline & Sensitivity & Specificity & Accuracy & AUC \\
\hline SWI-DWI mismatch & 46.7 & 55.6 & $63.0 \%$ & 0.511 \\
Standard PWI-DWI mismatch & 63.3 & 8.3 & $37.0 \%$ & 0.338 \\
Optimal PWI-DWI mismatch & 60.0 & 25.0 & $44.4 \%$ & 0.425 \\
Target PWI-DWI mismatch & 63.3 & 38.9 & $48.1 \%$ & 0.463 \\
\hline
\end{tabular}

Note:-AUC indicates area under the curve.

${ }^{a}$ Accuracy is the proportion of true results (both true-positives and true-negatives) in the population. Standard PWI-DWI mismatch was defined as PWI/DWI $\geq 1.2$, PWIDWI $\geq 10 \mathrm{~mL}$. Optimal PWI-DWI mismatch was defined as PWI/DWI $\geq 2.6$ and PWI-DWI $\geq 10 \mathrm{~mL}$. Target PWI-DWI mismatch was defined as PWI/DWI $\geq 1.2$, PWI$\mathrm{DWI} \geq 10 \mathrm{~mL}, \mathrm{PWI}$ (Tmax $\geq 8$ seconds) $\leq 100 \mathrm{~mL}$, and DWI $\leq 100 \mathrm{~mL}$.

signal change on SWI is mainly related to venous structures. ${ }^{1-14}$ In the setting of acute arterial occlusion, decreased oxygen supply causes an increased tissue oxygen extraction fraction, which leads to the increased level of deoxyhemoglobin and results in signal reduction of ipsilateral cerebral veins in the ischemic tissue on phase maps. ${ }^{8-10}$ Thus, as in the previous studies on gradient-echo T2* -weighted imaging, ${ }^{25,26}$ we used asymmetry of cerebral veins to reflect the change of oxygen extraction fraction in the ischemic hemisphere.

In the current study, we found that patients with a high cerebral vein AI score after ischemic stroke were more likely to have severe arterial occlusion and hypoperfusion. This outcome is consistent with that in a previous study which, by using gradient-echo $\mathrm{T} 2{ }^{\star}$-weighted imaging, demonstrated that abnormal prominent deep cerebral veins were correlated with a delayed MTT, a large perfusion defect, and increased CBV. ${ }^{9}$ Prominent superficial cerebral veins have also been correlated with an increase in CBV. ${ }^{15}$ Recently, an Alberta Stroke Program Early CT Score-based rating of asymmetric prominent vessels on SWI was shown to correlate best with MTT in patients with nonlacunar ischemic stroke within 24 hours after symptom onset. ${ }^{27}$ Therefore, extensive hypointense cerebral veins on SWI may be a useful indicator of ipsilateral large-artery occlusion and severe hypoperfusion. Most important, unlike the previous study, which found an association between abnormal visualization of the superficial venous system on gradient-echo $\mathrm{T} 2{ }^{\star}$-weighted imaging and smaller DWI lesions, ${ }^{15}$ we did not find such an association between the AI score and DWI lesion volume. According to previous literature, ${ }^{31,32}$ the superficial cerebral veins and deep cerebral veins differ in anatomic structure and function. ${ }^{25,33} \mathrm{We}$, therefore, considered it improper to compare the change of partial draining veins with DWI lesions.

We then tested the SWI-DWI mismatch, which may represent the presence of salvageable tissue based on tissue oxygen metabolism, and found that patients with SWI-DWI mismatch were

AJNR Am J Neuroradiol 35:2061-67 Nov 2014 www.ajnr.org 
more likely to benefit from thrombolytic therapy. One might argue that the relatively small DWI lesion volume in SWI-DWI mismatch may have a critical impact on patient outcome. However, we did not find a significant relationship between posttreatment neurologic outcome and either baseline DWI lesion volume or the AI score alone from logistic regression. Several studies have demonstrated metabolic derangement and evolution of infarction in brain regions with increased oxygen extraction fraction by PET. ${ }^{34,35}$ An increased oxygen extraction fraction may be a target for acute therapy to improve compromised cerebral circulation. Our study further revealed that in the presence of reperfusion, patients with SWI-DWI mismatch were more likely to have favorable outcome, compared with patients without it. We speculate that the presence of SWI-DWI mismatch after arterial occlusion in acute stroke may indicate salvageable tissue. The compensatory increase of oxygen uptake by tissue can provide the hypoxic tissue with a chance to revive if there is timely reperfusion. Therefore, we believe this MR imaging profile can identify the subgroup who may benefit from early reperfusion therapy. However, quantitative metabolic parameters that relate directly to the cerebral metabolic rate of oxygen consumption detected by PET are needed to assess this hypothesis.

The main advantage of using the SWI-DWI mismatch is that it provides a new concept to examine the penumbra on the basis of tissue oxygen metabolism. Although the SWI-DWI mismatch is not able to directly measure the cerebral metabolic rate of oxygen consumption, the concept of SWI-DWI mismatch is more closely aligned with the fundamental pathogenesis of neuronal energy disruption in acute ischemia than the perfusion-diffusion mismatch. In the perfusion-diffusion mismatch, identifying a perfusion threshold to reliably differentiate critically hypoperfused tissue $^{21}$ from benign oligemia, which will survive regardless of reperfusion, ${ }^{20,36}$ is a major challenge. The site of vascular occlusion and the extent of collateral blood flow also affect the complex relationship between perfusion lesions and their final fate. Another potential advantage of SWI over MR perfusion is that it obviates gadolinium contrast, which is contraindicated in patients with severe renal insufficiency or gadolinium allergy. ${ }^{37}$ The SWI-DWI mismatch may therefore be a useful alternative method that does not require MR perfusion to select patients who are good candidates for thrombolytic therapy. Moreover, our finding that patients with SWI-DWI mismatch achieved more favorable outcome based on successful reperfusion lends support to its future application in patients treated with an endovascular approach. In the current study, approximately $33 \%$ of patients had SWI-DWI mismatch. This was similar to the proportion (36\%) with "optimal" mismatch (mismatch ratio of $>2.6$ ) in post hoc analysis of the Diffusion and Perfusion Imaging Evaluation for Understanding Stroke Evolution study. ${ }^{20}$ These patients may be a suitable "responder" subgroup for randomization between reperfusion therapy and standard therapy.

There are limitations to our study. First, the exploratory nature of this study and the relatively small number of subjects necessitate the validation of the SWI-DWI mismatch model in an external dataset. Our conclusions should be considered hypothesis-generating and require further testing in prospective studies. Second, the asymmetry index of signal reduction along the cere- bral veins may vary among different MR imaging magnetic field strengths and can be influenced by the presence of microbleeds and a previous large ischemic stroke. Moreover, the segmentation of cerebral veins may be partly affected by cortex and ependymal epithelium signals. Third, the relationship of oxygen consumption and SWI-DWI mismatch needs further validation by PET studies. Fourth, the postprocessing and calculation of the AI index would take an additional 3 minutes, which may delay thrombolysis. Fifth, the definition of reperfusion and recanalization by MR imaging are still not unified. Last, our conclusions were all based on patients receiving tPA, without a control group, though we analyzed the outcome on the basis of reperfusion. Thus, we could not clarify whether withholding thrombolytic therapy in patients without a mismatch would yield better results. A randomized controlled trial would be needed to validate the usefulness of the SWI-DWI mismatch in the selection of patients for reperfusion therapy.

\section{CONCLUSIONS}

A high AI score of cerebral veins may be related to arterial occlusion or severe hypoperfusion. The presence of an SWI-DWI mismatch may predict a favorable outcome after intravenous thrombolysis. Our data provide evidence that SWI venography, related to oxygen metabolism, may provide an alternative for the evaluation of patients with acute ischemic stroke.

\section{ACKNOWLEDGMENTS}

We are grateful for the support from our patients. The authors thank Yi-hong Zhu, MD, from Zhejiang University School of Public Health for the critical verification of statistics, and Bruce Campbell, MD, from the Department of Neurology, Royal Melbourne Hospital, Australia, for the critical reading of the manuscript.

Disclosures: Min Lou—RELATED: grant support: Science Technology Department of Zhejiang Province (grant 2013C13G2010032), Zhejiang Provincial Natural Science Foundation (grant LRI2H09001), and the National Natural Science Foundation of China (grant 81171095).* * Money paid to the institution.

\section{REFERENCES}

1. Hacke W, Kaste M, Bluhmki E, et al. Thrombolysis with alteplase 3 to 4.5 hours after acute ischemic stroke. $N$ Engl J Med 2008;359:1317-29

2. Bivard A, Spratt N, Levi CR, et al. Acute stroke thrombolysis: time to dispense with the clock and move to tissue-based decision making? Expert Rev Cardiovasc Ther 2011;9:451-61

3. Grandin CB, Duprez TP, Smith AM, et al. Which MR-derived perfusion parameters are the best predictors of infarct growth in hyperacute stroke? Comparative study between relative and quantitative measurements. Radiology 2002;223:361-70

4. Heiss WD. The ischemic penumbra: correlates in imaging and implications for treatment of ischemic stroke: the Johann Jacob Wepfer award 2011. Cerebrovasc Dis 2011;32:307-20

5. Sobesky J. Refining the mismatch concept in acute stroke: lessons learned from PET and MRI. J Cereb Blood Flow Metab 2012;32:1416-25

6. Wang M, Dai Y, Han Y, et al. Susceptibility weighted imaging in detecting hemorrhage in acute cervical spinal cord injury. Magn Reson Imaging 2011;29:365-73

7. Haacke EM, Xu Y, Cheng YC, et al. Susceptibility weighted imaging (SWI). Magn Reson Med 2004;52:612-18 
8. An H, Lin W. Quantitative measurements of cerebral blood oxygen saturation using magnetic resonance imaging. J Cereb Blood Flow Metab 2000;20:1225-36

9. Hermier M, Nighoghossian N, Derex L, et al. Hypointense transcerebral veins at $\mathrm{T} 2^{\star}$-weighted MRI: a marker of hemorrhagic transformation risk in patients treated with intravenous tissue plasminogen activator. J Cereb Blood Flow Metab 2003;23:1362-70

10. Lee JM, Vo KD, An H, et al. Magnetic resonance cerebral metabolic rate of oxygen utilization in hyperacute stroke patients. Ann Neurol 2003;53:227-32

11. Heiss WD. Ischemic penumbra: evidence from functional imaging in man. J Cereb Blood Flow Metab 2000;20:1276-93

12. Powers WJ. Cerebral hemodynamics in ischemic cerebrovascular disease. Ann Neurol 1991;29:231-40

13. Robertson CA, McCabe C, Gallagher L, et al. Stroke penumbra defined by an MRI-based oxygen challenge technique. 2. Validation based on the consequences of reperfusion. J Cereb Blood Flow Metab 2011;31:1788-98

14. Santosh C, Brennan D, McCabe C, et al. Potential use of oxygen as a metabolic biosensor in combination with $\mathrm{T} 22^{*}$-weighted MRI to define the ischemic penumbra. J Cereb Blood Flow Metab 2008;28: 1742-53

15. Hermier M, Nighoghossian N, Derex L, et al. Hypointense leptomeningeal vessels at T2*-weighted MRI in acute ischemic stroke. $\mathrm{Neu}$ rology 2005;65:652-53

16. Davis SM, Donnan GA, Parsons MW, et al. Effects of alteplase beyond $3 \mathrm{~h}$ after stroke in the Echoplanar Imaging Thrombolytic Evaluation Trial (EPITHET): a placebo-controlled randomised trial. Lancet Neurol 2008;7:299-309

17. Jauch EC, Saver JL, Adams HP Jr, et al. Guidelines for the early management of patients with acute ischemic stroke: a guideline for healthcare professionals from the American Heart Association/ American Stroke Association. Stroke 2013;44:870-947

18. Cappellari M, Bovi P, Moretto G, et al. The THRombolysis and STatins (THRaST) study. Neurology 2013;80:655-61

19. Olivot JM, Mlynash M, Thijs VN, et al. Relationships between cerebral perfusion and reversibility of acute diffusion lesions in DEFUSE: insights from RADAR. Stroke 2009;40:1692-97

20. Kakuda W, Lansberg MG, Thijs VN, et al. Optimal definition for PWI/DWI mismatch in acute ischemic stroke patients. J Cereb Blood Flow Metab 2008;28:887-91

21. Olivot JM, Mlynash M, Thijs VN, et al. Optimal Tmax threshold for predicting penumbral tissue in acute stroke. Stroke 2009;40:469-75

22. Lansberg MG, Straka M, Kemp S, et al. MRI profile and response to endovascular reperfusion after stroke (DEFUSE 2): a prospective cohort study. Lancet Neurol 2012;11:860-67

23. Straka M, Albers GW, Bammer R. Real-time diffusion-perfusion mismatch analysis in acute stroke. J Magn Reson Imaging 2010;32: 1024-37

24. Fiebach JB, Al-Rawi Y, Wintermark M, et al. Vascular occlusion enables selecting acute ischemic stroke patients for treatment with desmoteplase. Stroke 2012;43:1561-66

25. Jensen-Kondering U, Böhm R. Asymmetrically hypointense veins on $\mathrm{T} 2{ }^{\star} \mathrm{W}$ imaging and susceptibility-weighted imaging in ischemic stroke. World J Radiol 2013;5:156-65

26. Rosso C, Belleville M, Pires C, et al. Clinical usefulness of the visibility of the transcerebral veins at $3 \mathrm{~T}$ on $\mathrm{T} 2^{\star}$-weighted sequence in acute stroke patients. Eur J Radiol 2012;81:1282-87

27. Kao HW, Tsai FY, Hasso AN. Predicting stroke evolution: comparison of susceptibility-weighted MR imaging with MR perfusion. Eur Radiol 2012;22:1397-403

28. Lansberg MG, Thijs VN, Bammer R, et al. The MRA-DWI mismatch identifies patients with stroke who are likely to benefit from reperfusion. Stroke 2008;39:2491-96

29. Parsons MW, Christensen S, McElduff P, et al. Pretreatment diffusion- and perfusion-MR lesion volumes have a crucial influence on clinical response to stroke thrombolysis. J Cereb Blood Flow Metab 2010;30:1214-25

30. Albers GW, Thijs VN, Wechsler L, et al. Magnetic resonance imaging profiles predict clinical response to early reperfusion: the diffusion and perfusion imaging evaluation for understanding stroke evolution (DEFUSE) study. Ann Neurol 2006;60:508-17

31. Sheerin F. The imaging of the cerebral venous sinuses. Semin Ultrasound CT MR 2009;30:525-58

32. Leach JL, Fortuna RB, Jones BV, et al. Imaging of cerebral venous thrombosis: current techniques, spectrum of findings, and diagnostic pitfalls. Radiographics 2006;26(suppl 1):S19-41, discussion S42-43

33. Oran I, Memis A, Sener RN, et al. The so-called transcerebral veins: appearance in three different cases. Comput Med Imaging Graph 1999;23:127-31

34. Hakim AM, Evans AC, Berger L, et al. The effect of nimodipine on the evolution of human cerebral infarction studied by PET. J Cereb Blood Flow Metab 1989;9:523-34

35. Wise RJ, Bernardi S, Frackowiak RS, et al. Serial observations on the pathophysiology of acute stroke: the transition from ischaemia to infarction as reflected in regional oxygen extraction. Brain 1983; 106(pt 1):197-222

36. Kidwell CS, Alger JR, Saver JL. Beyond mismatch: evolving paradigms in imaging the ischemic penumbra with multimodal magnetic resonance imaging. Stroke 2003;34:2729-35

37. Marckmann P, Skov L, Rossen K, et al. Nephrogenic systemic fibrosis: suspected causative role of gadodiamide used for contrastenhanced magnetic resonance imaging. J Am Soc Nephrol 2006;17: 2359-62 